\title{
Corrigendum
}

\section{Corrigendum to "Decay Experiments of Effective N-Removing Microbial Communities in Sequencing Batch Reactors"}

\author{
Chen Lv, ${ }^{1,2,3}$ Li Ming, ${ }^{2}$ Shuang Zhong, ${ }^{2}$ Jianlong Wang $\left(\mathbb{D},{ }^{1}\right.$ Wu Lei, ${ }^{2}$ A. Gizem Mutlu, ${ }^{3}$ \\ and Barth F. Smets ${ }^{3}$ \\ ${ }^{1}$ Laboratory of Environmental Technology, Institute of Nuclear and New Energy Technology, Tsinghua University, \\ Beijing 100084, China \\ ${ }^{2}$ Key Laboratory of Songliao Aquatic Environment, Ministry of Education, Jilin Jianzhu University, Changchun 130118, \\ China \\ ${ }^{3}$ Department of Environmental Engineering, Technical University of Denmark, 2800 Kongens Lyngby, Denmark
}

Correspondence should be addressed to Jianlong Wang; wangjl@mail.tsinghua.edu.cn

Received 3 May 2018; Accepted 23 May 2018; Published 9 August 2018

Copyright ( $\odot 2018$ Chen Lv et al. This is an open access article distributed under the Creative Commons Attribution License, which permits unrestricted use, distribution, and reproduction in any medium, provided the original work is properly cited.

In the article titled "Decay Experiments of Effective N-Removing Microbial Communities in Sequencing Batch Reactors" [1], Professor Dr. Barth F. Smets and Dr. A. Gizem Mutlu were missing from the authors' list. Professor Smets was fully involved in the planning and design of the study, as well as the interpretation of the results, and he revised an earlier report of this research. Dr. Mutlu was fully involved in the experimental design, conceptualization, and methodology, provided the initial biological material for the decay experiments, and provided substantial guidance throughout the decay experiments and data collection, as well as analysis of the data, interpretation of the results, and presentation of the data in an earlier report of these results. Additionally, there was a missing affiliation for the first author. The corrected authors' list and affiliations are shown above.

\section{References}

[1] C. Lv, M. Li, S. Zhong, J. Wang, and L. Wu, "Decay experiments of effective N-removing microbial communities in sequencing batch reactors," Journal of Chemistry, vol. 2017, Article ID 4878910, 5 pages, 2017. 

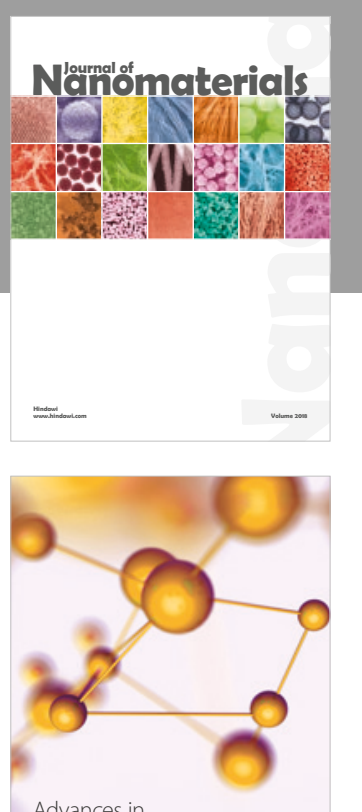

Physical Chemistry
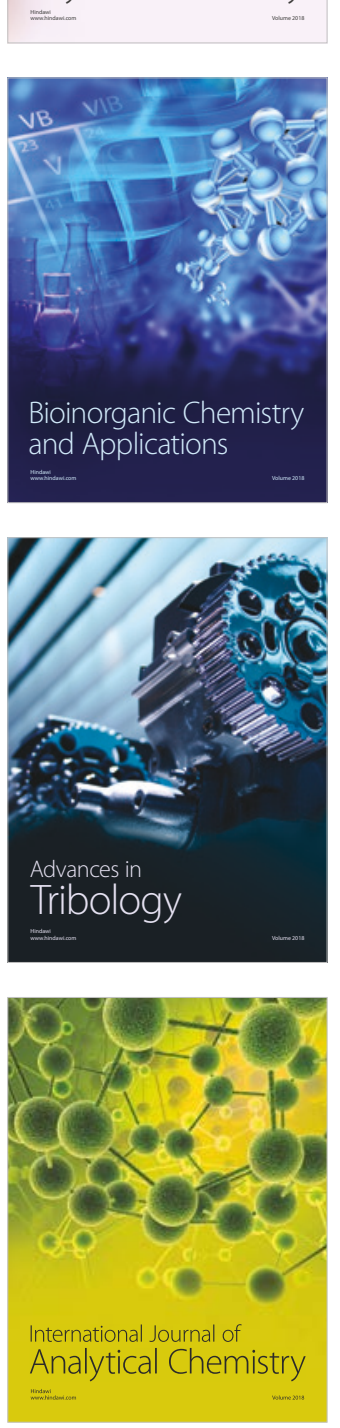

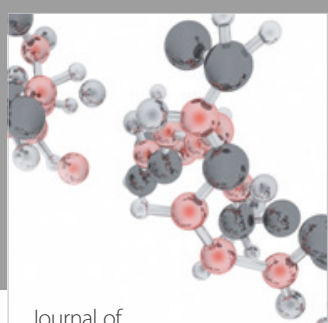

Analytical Methods

in Chemistry

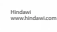

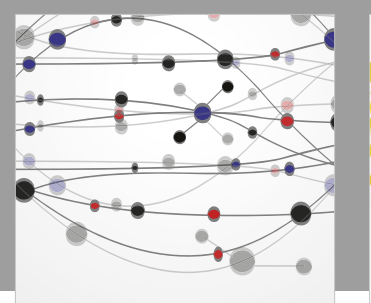

The Scientific World Journal

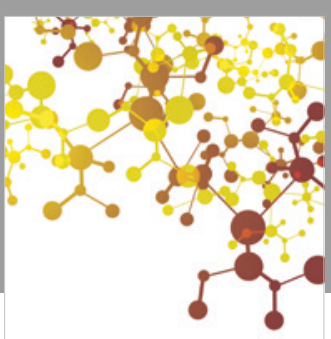

Journal of

Applied Chemistry
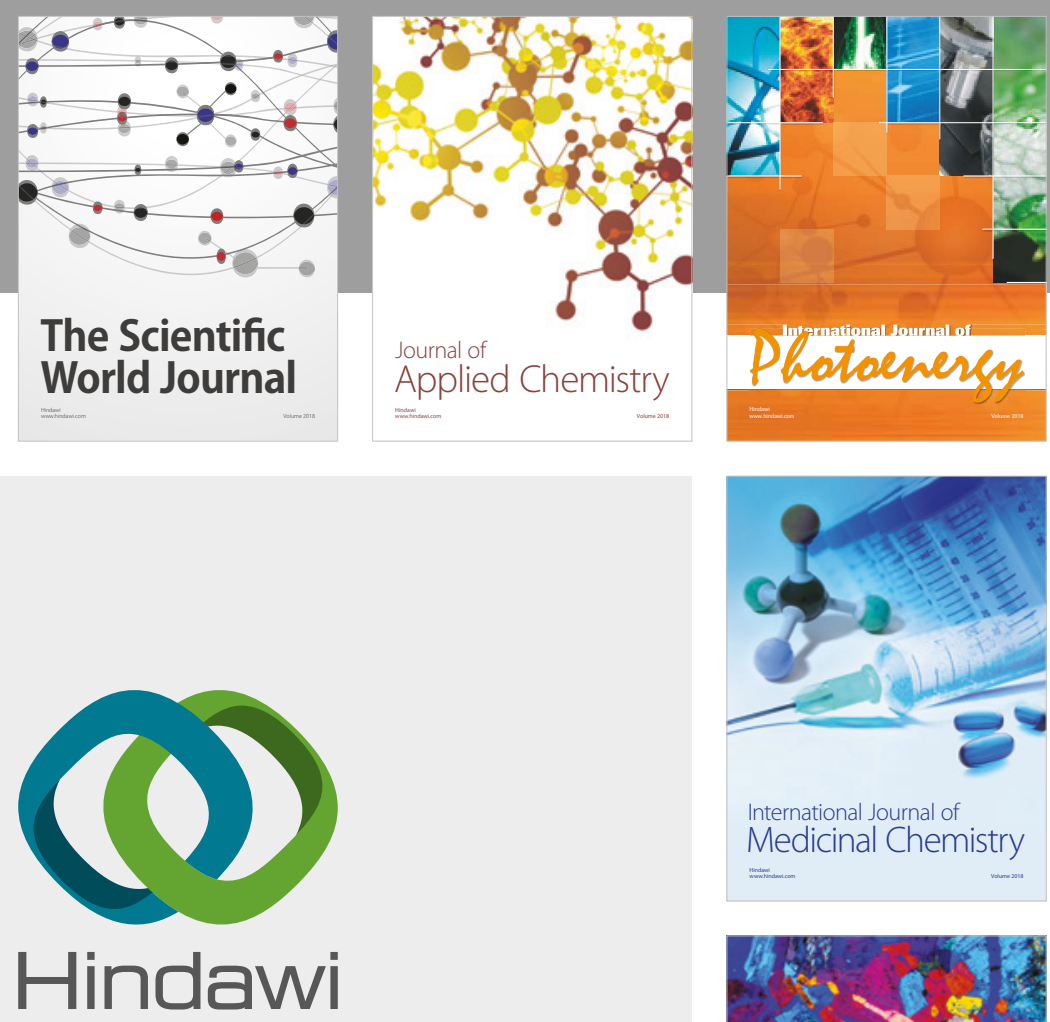

Submit your manuscripts at

www.hindawi.com
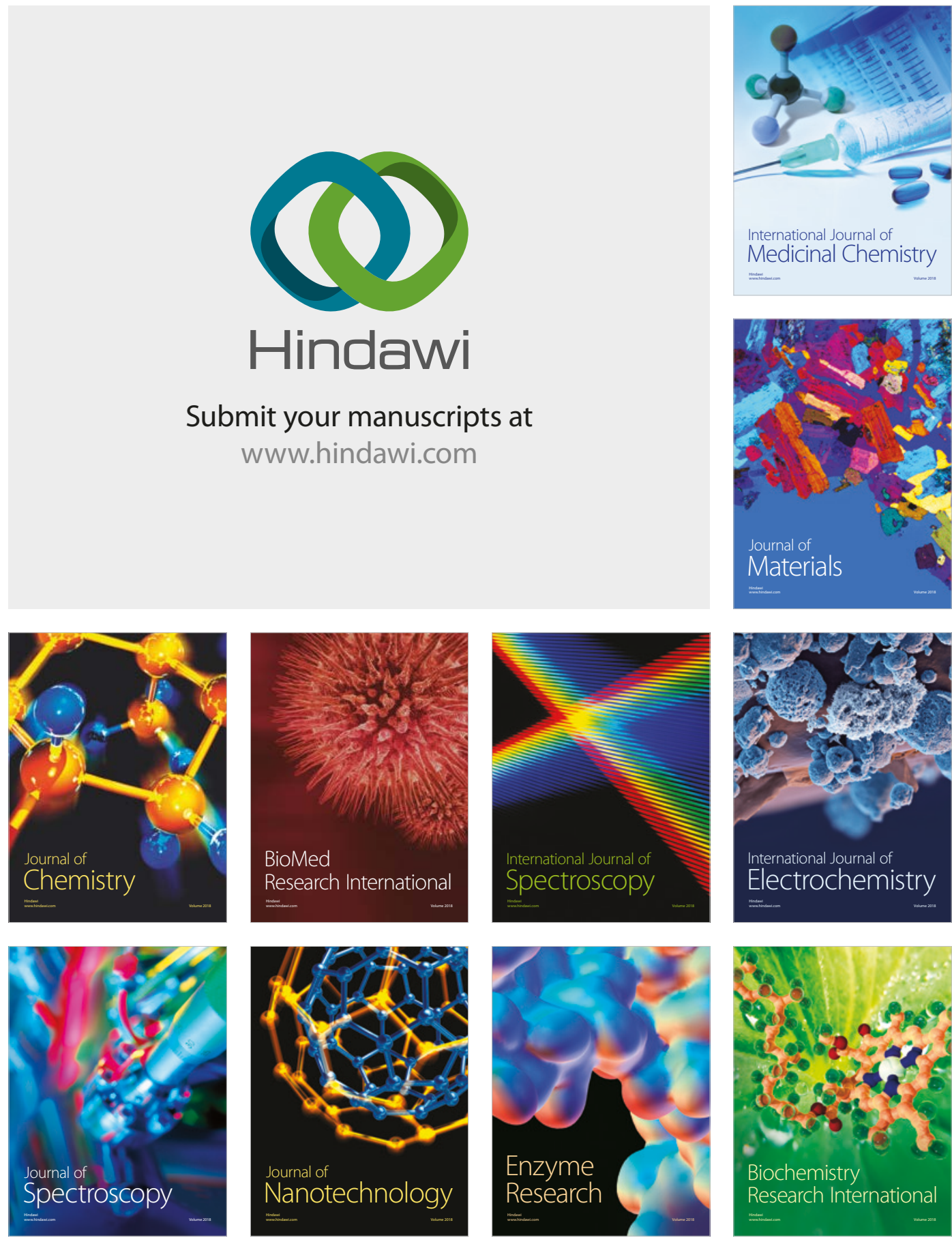
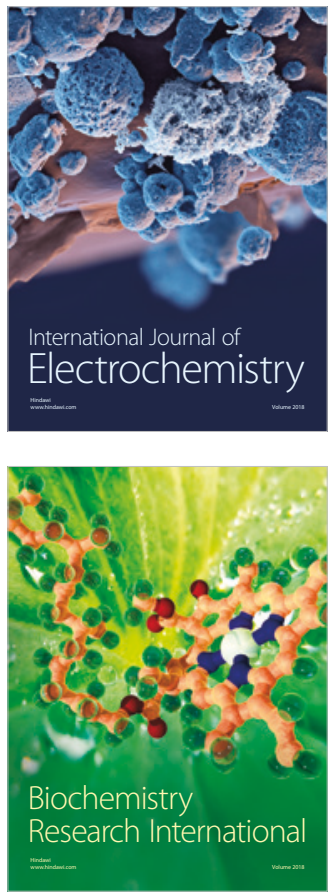